\title{
Fast-Track Extubation in Pediatric Cardiothoracic Surgery in Developing Countries
}

\author{
${ }^{1}$ Department of Paediatric and Congenital Cardiac Surgery and \\ Cardiology, Azienda Ospedaliero-Universitaria, Ancona, Italy \\ 2 Department of Cardiothoracic and Vascular Surgery, Kenyatta \\ National Hospital and University of Nairobi, Nairobi, Kenya \\ ${ }^{3}$ Department of Anesthesia and Critical Care, Heart hospital \\ "G. Pasquinucci," Fondazione Toscana Gabriele Monasterio, \\ Massa, Italy \\ J Card Crit Care TSS 2017;1:21-23.
}

Federica lezzi ${ }^{1}$ Michele di Summa ${ }^{2}$ Paolo Del Sarto ${ }^{3}$ James Munene ${ }^{2}$

\author{
Address for correspondence Federica lezzi, MD, Department of \\ Paediatric and Congenital Cardiac Surgery and Cardiology, Azienda \\ Ospedaliero-Universitaria Ospedali Riuniti Ancona "Umberto I, G. M. \\ Lancisi, G. Salesi," via Conca n.71, 60128 Ancona, Italy \\ (e-mail: federicaiezzi@hotmail.it).
}

\begin{abstract}
Keywords

- congenital heart disease

- fast tracking

- mechanical ventilation

Objective In recent years, low-dose, short-acting anesthetic agents, which replaced the former high-dose opioid regimens, offer a faster postoperative recovery and decrease the need for mechanical ventilatory support. In this study, the aim was to determine the success rate of fast-track approach in surgical procedures for congenital heart disease.

Methods There is some evidence, mostly from retrospective analyses, that fast tracking can be beneficial. Ninety-one cases with moderate complex cardiac malformations were operated with fast-track protocol during cardiothoracic charitable missions. The essential aspects of early extubation in our cohort included selected patients with good preoperative status, good surgical result with hemodynamic stability in low dose of inotropic drugs at the end of bypass, and no active bleeding. In this setting, a careful choice and dosing of anesthetic agents, alongside a good postoperative analgesia are mandatory.

Results The authors found that an early extubation ( $<4$ hours) can be both effective and safe as it reduces intubation and ventilator times without increasing postoperative complications in pediatric congenital heart disease.

Conclusion This study supports a wider use of fast-track extubation protocols in pediatric patients submitted for congenital cardiac surgery in developing countries.
\end{abstract}

\section{Introduction}

In recent years, earlier weaning from mechanical ventilation has been emphasized more for postoperative intensive care for children with congenital heart disease. Fast tracking in cardiac surgery refers to the concept of early extubation, mobilization, and hospital discharge in an effort to reduce the cost and perioperative morbidity. ${ }^{1}$

Potential advantages of fast tracking following surgery for congenital heart disease include reduced ventilator-associated complications, reduced requirements of sedatives, rapid patient mobilization, earlier intensive care unit (ICU) discharges, and decreased length of hospital stay. Fast track- ing is not restricted to anesthetic management; it is made possible by using effective multidisciplinary patient management strategies.

Early extubation after pediatric open cardiac surgery, as a part of a fast-track strategy, is still not a common practice. Several more recent studies describing early extubation in children have included only simple surgical repairs in relatively low-risk patients. Very early extubation refers to planned extubation either in the operating room or immediately upon arrival in the ICU. ${ }^{2}$

Settings in surgery for congenital heart disease in which fast tracking cannot be considered safe include all patients who are hemodynamically unstable, coagulopathic patients,
DOI https://doi.org/ $10.1055 / \mathrm{s}-0037-1604334$.
Copyright ( 2017 Official Publication of License terms The Simulation Society (TSS), accredited by International Society of Cardiovascular Ultrasound (ISCU) 
have coagulopathy or patients who do not meet generally accepted extubation criteria. $^{3}$

Shortening of both postoperative ventilation and ICU length of stay is an important goal to avoid complications related to mechanical ventilation, improve patient outcome, and reduce costs of intensive care treatment.

The authors describe their experience of postoperative fast tracking of children who underwent cardiac surgery, during charitable missions in developing countries where resources and equipment were severely limited.

\section{Materials and Methods}

During humanitarian cardiothoracic surgical missions in Kenyatta National Hospital (Nairobi, Kenya), both male and female patients aged 4 months to 18 years, undergoing elective surgical procedures on cardiopulmonary bypass or off-pump for congenital heart disease, were included in the study.

From December 2013 to April 2016, closed cardiac operations were performed in 19 (20.8\%) patients, which included modified Blalock-Taussig shunt, pulmonary artery banding, patent ductus arteriosus closure, aortic coarctation, and Glenn procedure.

In addition, 72 (79.2\%) patients underwent cardiopulmonary bypass. This group included ventricular septal defect, atrial septal defect, tetralogy of Fallot, and atrioventricular septal defect.

After median sternotomy and heart exposure, a dosage of heparin $300 \mathrm{UI} / \mathrm{kg}$ was given to obtain an activated clotting time (ACT $>480$ seconds, vessels cannulation was performed, and cardiopulmonary bypass was performed under moderate hypothermia at $32^{\circ} \mathrm{C}$.

Cold crystalloid St. Thomas cardioplegia was administered in the aorta after clamping, with induction dosage of 30 $\mathrm{mL} / \mathrm{kg}$ and repeated every 30 minutes with dosage of $20 \mathrm{~mL} /$ $\mathrm{kg}$. The hematocrit level during cardiopulmonary bypass was maintained $>24 \%$. In all the open-heart cases, the authors used continuous ultrafiltration during cardiopulmonary bypass time.

The essential aspects of early extubation in this cohort included selected patients with good preoperative status, good surgical result with hemodynamic stability in low dose of inotropic drugs at the end of bypass, and no active bleeding. In this setting, a careful choice and dosage of anesthetic agents, alongside a good postoperative analgesia, are mandatory.

The patients were induced with intravenous midazolam $0.1 \mathrm{mg} / \mathrm{kg}$, fentanyl 5 to $10 \mu \mathrm{g} / \mathrm{kg}$, and pancuronium $0.1 \mathrm{mg} / \mathrm{kg}$. Maintenance anesthesia consisted of boluses of low-dose fentanyl at 1 to $2 \mu \mathrm{g} / \mathrm{kg}$ (total dose $<12.5 \mu \mathrm{g} / \mathrm{kg}$ ) along with boluses of midazolam at $0.1 \mathrm{mg} / \mathrm{kg}$ at the beginning of cardiopulmonary bypass and small dose of halogenated agents (isoflurane 0.8-1 MAC, minimum alveolar concentration). Since November 2014, the anesthesia plan has changed with the introduction of remifentanil $0.15-0.25 \mu \mathrm{g} / \mathrm{kg} / \mathrm{min}$ plus propofol infusion of 2 to $5 \mathrm{mg} / \mathrm{kg} / \mathrm{h}$ as maintenance, along with midazolam at $0.1 \mathrm{mg} / \mathrm{kg}$ and propofol infusion of
2 to $5 \mathrm{mg} / \mathrm{kg} / \mathrm{h}$ and small dose of halogenated agents (isoflurane 0.8-1 MAC, minimum alveolar concentration). Atracurium in a repeated dose of $0.5 \mathrm{mg} / \mathrm{kg}$ intravenously was introduced as muscle relaxant. Remifentanil and propofol drip combined with short-acting anesthetic adjuvants facilitated early awakening and tracheal extubation after surgery.

For analgesia, adjuvants intravenous paracetamol $15 \mathrm{mg} / \mathrm{kg}$ and ketorolac $0.5 \mathrm{mg} / \mathrm{kg}$ were administered before chest closure. In a remifentanil-based anesthesia, a bolus dose of morphine, 0.1 to $0.15 \mathrm{mg} / \mathrm{kg}$, was administered at the sternum closure. At skin closure, bupivacaine $2.5 \mathrm{mg} / \mathrm{kg}$ diluted in saline was infiltrated at the wound and drain sites.

If necessary, opioids were reversed after stabilization of the hemodynamic status, in ICU. Supplementation with hypnotic drugs allows reduction in the opioid dose and enabling earlier extubation without compromising hemodynamic stability.

Extubation criteria included fully awake normothermic patients (core temperature $36^{\circ} \mathrm{C}$ ) with regular breathing and tidal volume of 5 to $7 \mathrm{~mL} / \mathrm{kg}$, normal preextubation arterial blood gas analysis on $\mathrm{FiO}_{2}$ of 0.4 , optimal hemoglobin with no metabolic acidosis, stable hemodynamics with minimal inotropic support, minimal chest tube drainage, and normal perioperative post-repair transesophageal or epicardial echocardiography.

The patients undergoing atrial septal defect and patent ductus arteriosus closure, Glenn shunt, and aortic coarctation were assessed to be extubated directly in operating theater. The patients undergoing other procedures mentioned were assessed for extubation and extubated within 4 hours of arrival in ICU.

\section{Results}

The median duration of mechanical ventilation was 2.85 hours. The incidence of postoperative complications, including pneumonia and atelectasis (8\%), organ failure (1\%), and reintubation (3\%), was significantly low. The reasons for reintubation rate were hemodynamic instability and respiratory complication.

Of the total 91 children and young adult included in the study, 36 (39.5\%) patients were extubated in operating theater, $16(17.6 \%)$ were extubated within 1 hour of arrival in ICU, and 39 (42.9\%) were extubated within 4 hours of arrival in ICU.

The Venturi mask at $50 \%$ is usually applied after extubation, with oxygen flow at 8 to $10 \mathrm{~L} / \mathrm{min}$. Its use is suspended after the arterial gasometry analysis, allowing the progression to nasal oxygen and using a spectacle-type nasal catheter holder and, afterward, natural air. The patients are discharged from the ICU only if their oxygenation is adequate without providing additional oxygen.

The ICU internment ranged from 1 to 4 days and the hospital internment from 5 to 10 days.

The results of fast-track and delayed extubation calculated as percentages with respect to sex and weight were not clinically significant ( $p \geq 0.05$ ). The study results validated 
the safety of fast-track extubation in patients undergoing pediatric congenital heart disease surgery at this pediatric cardiac surgery setup.

\section{Discussion}

Fast tracking in congenital heart surgery is not uniformly accepted, although it was introduced as early as the late 1970s. Large multicenter, controlled studies confirming potential benefits have not been performed; thus, concerns about the safety of such an approach remain, despite numerous reports about the feasibility of fast tracking in the pediatric cardiac surgery population. ${ }^{4}$

Studies show that early extubation of elective cardiac surgery patients does not increase perioperative morbidity. The introduction of improved and new anesthetic agents such as modern inhalational anesthetics, short-acting opioids, hypnotics, and sedatives with favorable pharmacodynamics and kinetic profiles are safe and make possible an anesthesia plan open toward the possibility of an early extubation and mobilization at the end of surgery.

With the development of short acting-anesthetics, opioids, and inhalational anesthetics with less cardiac depressive effects, early extubation after pediatric cardiothoracic operations can be safely achieved in selected patients.

Aside from anesthesia management, essential components for the safe execution of the system would be to moderate simple correction procedures with good surgical result, minimally invasive approaches, cardiopulmonary bypass management, and postoperative ICU care. Ultrafiltration strategies to reduce the inflammatory response commonly seen in open-heart surgery as continuous ultrafiltration during prolonged cardiopulmonary bypass or modified ultrafiltration at the end are an important part of this fast-track management. Ultrafiltration strategies and a good surgical hemostatic control can influence fast-tracking: a higher level of hematocrit beside a better fluid balance control, reducing the need of transfusion, and improving the pulmonary function at the end of surgical procedures.

On the basis of experience from the current study, all the mentioned advantages were achieved including better patient outcome and best possible resource utilization. There was an improvement in patient turn over from ICU and improved ability to treat more patients with congenital heart disease. $^{5}$

It was demonstrated that early extubation (within 4 hours) in children undergoing surgery for congenital heart disease had no negative affect on cardiac function. ${ }^{6}$

The rate of reintubation following early extubation with modern anesthetics is low and mostly unrelated to fast tracking. The authors attribute this to improved surgical techniques, cardiopulmonary bypass refinements such as minimal priming volumes, the use of ultrafiltration technique, and the availability of drugs with favorable pharmacokinetics and pharmacodynamics.

In conclusion, fast-track protocols offer the opportunity of earlier extubation of the patients with noncomplex cardiac anomalies and may result in spending fewer hours in ICU. The hospital stay also decreases and patients can be discharged earlier in many cases.

\section{Conflict of Interest}

None.

\section{References}

1 Neirotti RA, Jones D, Hackbarth R, Paxson Fosse G. Early extubation in congenital heart surgery. Heart Lung Circ 2002;11(03):157-161

2 Lake CL. Fast tracking in paediatric cardiac anaesthesia: an update. Ann Card Anaesth 2002;5(02):203-208

3 Miller JW, Vu D, Chai PJ, et al. Patient and procedural characteristics for successful and failed immediate tracheal extubation in the operating room following cardiac surgery in infancy. Paediatr Anaesth 2014;24(08):830-839

4 DiNardo JA. Con: extubation in the operating room following pediatric cardiac surgery. J Cardiothorac Vasc Anesth 2011; 25(05):877-879

5 Mittnacht AJC, Hollinger I. Fast-tracking in pediatric cardiac surgery-the current standing. Ann Card Anaesth 2010;13(02): 92-101

6 Meissner U, Scharf J, Dötsch J, Schroth M. Very early extubation after open-heart surgery in children does not influence cardiac function. Pediatr Cardiol 2008;29(02):317-320 